\title{
ON THE NONABELIAN TENSOR SQUARE OF A NILPOTENT GROUP OF CLASS TWO
}

\author{
by MICHAEL R. BACON
}

(Received 18 December, 1992)

1. Introduction. The nonabelian tensor square $G \otimes G$ of a group $G$ is generated by the symbols $g \otimes h, g, h \in G$, subject to the relations

$$
g g^{\prime} \otimes h=\left({ }^{g} g^{\prime} \otimes{ }^{g} h\right)(g \otimes h) \quad \text { and } \quad g \otimes h h^{\prime}=(g \otimes h)\left({ }^{h} g \otimes{ }^{h} h^{\prime}\right),
$$

for all $g, g^{\prime}, h, h^{\prime} \in G$, where ${ }^{g} g^{\prime}=g g^{\prime} g^{-1}$. The tensor square is a special case of the nonabelian tensor product which has its origins in homotopy theory. It was introduced by R. Brown and J. L. Loday in [4] and [5], extending ideas of Whitehead in [6].

In their seminal paper [3], R. Brown, D. J. Johnson, and E. F. Robertson raise the question if a general estimate can be given for $d(G \otimes G)$ in terms of $d(G)$, where $d(G)$ is the minimal number of generators for a group $G$. The topic of this paper is to give an estimate for $d(G \otimes G)$ in terms of $d(G)$ in case $G$ is nilpotent of class 2 (Theorem 3.1). For a group being free nilpotent of class 2 we show this estimate is sharp (Theorem 3.3). This is done by explicitly determining the nonabelian tensor square of a free nilpotent group of class 2 on $n$ generators as a free abelian group of suitable rank. In case $n=2, \mathrm{R}$. Aboughazi in [1] obtained the result using a different method.

Based on results in [3], it follows that the tensor square of a nilpotent group is nilpotent, where $\operatorname{cl}(G \otimes G)=\operatorname{cl}\left(G^{\prime}\right)$ or $\operatorname{cl}\left(G^{\prime}\right)+1$. We will show that for a group $G$ of class 2 , the tensor square $G \otimes G$ is always abelian (Proposition 2.2). This fact enables us to use the concept of a crossed pairing in our computations. We define it here in the case relevant for tensor squares. For the general case of a nonabelian tensor product we refer to $[3]$.

Definition 1.1. Let $G$ and $L$ be groups. A function $\phi: G \times G \rightarrow L$ is called a crossed pairing if

$$
\begin{aligned}
& \phi\left(g g^{\prime}, h\right)=\phi\left({ }^{g} g^{\prime},{ }^{g} h\right) \phi(g, h), \\
& \phi\left(g, h h^{\prime}\right)=\phi(g, h) \phi\left({ }^{h} g,{ }^{h} h^{\prime}\right)
\end{aligned}
$$

for all $g, g^{\prime}, h, h^{\prime} \in G$.

Crossed pairings allow us to determine homomorphic images of $G \otimes G$ as follows.

Proposition 1.2 [3]. A crossed pairing $\phi$ determines a unique homomorphism of groups $\phi^{*}: G \otimes G \rightarrow L$ such that $\phi^{*}(g \otimes h)=\phi(g, h)$ for all $g, h \in G$.

The fact that $G \otimes G$ is abelian in our case allows us to use crossed pairings explicitly in our computations. In [2] this method has been used to determine the nonabelian tensor square of all 2-generator $p$-groups of class 2, where $p$ is an odd prime. Until then it seems that crossed pairings have only been applied in theoretical context, e.g. Proposition 7 in [3]. In cases where $G \otimes G$ fails to be commutative, the extensive calculations involved and conjecturing the group $L$ pose an obstacle to using this method.

Glasgow Math. J. 36 (1994) 291-296. 
2. Basic results. It follows from general results in [3] that $\operatorname{cl}(G \otimes G) \leq 2$ if $\operatorname{cl}(G)=2$. We are able to lower this bound. This is fundamental in establishing the desired estimates for $d(G \otimes G)$ if $\operatorname{cl}(G)=2$.

The following familiar expansion formulas for groups of class 2 are stated here without proof and will be used without further reference. $n \in \mathbb{Z}$,

LEMMA 2.1. Let $G$ be a group of nilpotency class two. Then, for any $a, b \in G$ and any

$$
\left[a, b^{n}\right]=\left[a^{n}, b\right]=[a, b]^{n} \quad \text { and } \quad(a b)^{n}=a^{n} b^{n}[b, a]^{\left(\frac{n}{2}\right)} .
$$

The first results now follow.

Proposition 2.2. If $G$ is a nilpotent group of class two, then $G \otimes G$ is abelian and $1_{\otimes}=[x, y] \otimes\left[x^{\prime}, y^{\prime}\right]$ for all $x, y, x^{\prime}, y^{\prime} \in G$.

Proof. By Proposition 3 in [3], we have

$$
(x \otimes y)\left(x^{\prime} \otimes y^{\prime}\right)(x \otimes y)^{-1}=\left({ }^{[x, y]} x^{\prime} \otimes{ }^{[x, y]} y^{\prime}\right)=\left(x^{\prime} \otimes y^{\prime}\right),
$$

since $G^{\prime}$ is central in $G$, and hence $\left[x \otimes y, x^{\prime} \otimes y^{\prime}\right]=1_{\otimes}$. Thus $G \otimes G$ is abelian. Again by Proposition 3 in $[3]$ we have $\left[x \otimes y, x^{\prime} \otimes y^{\prime}\right]=[x, y] \otimes\left[x^{\prime}, y^{\prime}\right]$; thus $[x, y] \otimes\left[x^{\prime}, y^{\prime}\right]=1_{\otimes}$.

Proposition 2.3. Let $G$ be a group of nilpotency class 2 . Then the defining relations of $G \otimes G$ reduce to

$$
\begin{aligned}
& x x^{\prime} \otimes y=(x \otimes y)\left(x^{\prime} \otimes y\right)\left(\left[x, x^{\prime}\right] \otimes y\right)\left(x^{\prime} \otimes[x, y]\right), \\
& x \otimes y y^{\prime}=(x \otimes y)\left(x \otimes y^{\prime}\right)\left(x \otimes\left[y, y^{\prime}\right]\right)\left([y, x] \otimes y^{\prime}\right)
\end{aligned}
$$

for all $x, x^{\prime}, y, y^{\prime} \in G$.

Proof. Let $x, x^{\prime}, y, y^{\prime}$ be any elements in $G$, where $\operatorname{cl}(G)=2$. By the defining relations for the tensor square and Proposition 2.2, we obtain

$$
\begin{aligned}
x x^{\prime} \otimes y & =\left({ }^{x} x^{\prime} \otimes{ }^{x} y\right)(x \otimes y)=\left(\left[x, x^{\prime}\right] x^{\prime} \otimes[x, y] y\right)(x \otimes y) \\
& =\left(x^{\prime} \otimes y\right)\left(\left[x, x^{\prime}\right] \otimes[x, y]\right)\left(\left[x, x^{\prime}\right] \otimes y\right)\left(x^{\prime} \otimes[x, y]\right)(x \otimes y) \\
& =\left(x^{\prime} \otimes y\right)\left(\left[x, x^{\prime}\right] \otimes y\right)\left(x^{\prime} \otimes[x, y]\right)(x \otimes y) .
\end{aligned}
$$

Thus (2.3.1) holds. Similarly, by expanding $x \otimes y y^{\prime}$ we obtain (2.3.2).

As a consequence of Proposition 2.3 we have the following results.

COROLlaRY 2.4. If $G$ is a group of nilpotency class two, then the following relations hold for all $x, y, z \in G$ :

$$
\begin{gathered}
([x, y] \otimes z)(y \otimes[x, z])(x \otimes[z, y])=1_{\otimes}, \\
(x \otimes[y, z])([x, z] \otimes y)([y, x] \otimes z)=1_{\otimes}, \\
([x, y] \otimes z)=(z \otimes[x, y])^{-1} .
\end{gathered}
$$

Proof. Observe that $x y=[x, y] y x$ in any group. Consider the element $x y \otimes z \in$ $G \otimes G$, where $\operatorname{cl}(G)=2$. Expand first as

$$
x y \otimes z=(x \otimes z)(y \otimes z)([x, y] \otimes z)(y \otimes[x, z])
$$


by Proposition 2.3, and secondly as

$$
\begin{aligned}
x y \otimes z=[x, y] y x \otimes z & =([x, y] \otimes z)(y x \otimes z) \\
& =([x, y] \otimes z)(x \otimes z)(y \otimes z)([y, x] \otimes z)(x \otimes[y, z]) \\
& =(x \otimes z)(y \otimes z)(x \otimes[y, z]) .
\end{aligned}
$$

Equating the terms from the two expansions yields

$$
([x, y] \otimes z)(y \otimes[x, z])=(x \otimes[y, z])
$$

Note that by Proposition 3 in [3] we have $(x \otimes[y, z])^{-1}=(x \otimes[z, y])$ and multiplying both sides by $x \otimes[z, y]$ yields (2.4.1). Similarly, expanding $x \otimes y z$ in the same fashion as above, we obtain

$$
(x \otimes[y, z])([y, x] \otimes z)=([z, x] \otimes y) .
$$

Multiplying both sides by $([x, z] \otimes y)$ yields (2.4.2). In order to derive the last relation, multiplying (2.4.1) and (2.4.2) together yields

$$
\begin{aligned}
1_{\otimes} & =(x \otimes[y, z])([x, z] \otimes y)([y, x] \otimes z)([x, y] \otimes z)(y \otimes(x, z])(x \otimes[z, y]) \\
& =(x \otimes[y, z])(x \otimes[y, z])^{-1}([y, x] \otimes z)([y, x] \otimes z)^{-1}([x, z] \otimes y)(y \otimes[x, z]) \\
& =([x, z] \otimes y)(y \otimes[x, z]) .
\end{aligned}
$$

To establish our primary result we shall need the following technical lemmas.

Lemma 2.5. Let $G$ be a group of nilpotency class two. Then: for $x, y_{1}, \ldots, y_{n} \in G$,

$$
x \otimes \prod_{i=1}^{n} y_{i}=\prod_{i=1}^{n}\left(x \otimes y_{i}\right) \prod_{i=2}^{n} \prod_{j=1}^{i-1}\left(\left[y_{i}, x\right] \otimes y_{j}\right)
$$

for $y, x_{1}, \ldots, x_{n} \in G$

$$
\prod_{i=1}^{n} x_{i} \otimes y=\prod_{i=1}^{n}\left(x_{i} \otimes y\right) \prod_{i=2}^{n} \prod_{j=1}^{i-1}\left(x_{j} \otimes\left[x_{i}, y\right]\right)
$$

for $x_{1}, \ldots, x_{n}, y_{1}, \ldots, y_{n} \in G$,

$$
\prod_{i=1}^{n} x_{i} \otimes \prod_{j=1}^{m} y_{j}=\prod_{i=1}^{n} \prod_{j=1}^{m}\left(x_{i} \otimes y_{j}\right) \cdot M
$$

with

$$
M=\prod_{i=1}^{n} \prod_{j=2}^{m} \prod_{k=1}^{j-1}\left(\left[y_{j}, x_{i}\right] \otimes y_{k}\right) \prod_{i=2}^{n} \prod_{k=1}^{i-1} \prod_{j=1}^{m}\left(x_{k} \otimes\left[x_{i}, y_{j}\right]\right),
$$

where in each case the empty product is interpreted as the identity.

Proof. The proof of (2.5.1) and (2.5.2) is by induction on $n$ using Proposition 2.2 and 2.3 as well as Corollary 2.4 . The expansion of (2.5.3) is obtained by combining (2.5.1) and (2.5.2). The details are omitted.

The next lemma is an immediate consequence of (2.4.3) and (2.5.3).

LEMMA 2.6. Let $G$ be a group of nilpotency class two, then for any $x, y \in G$ and any integers $m$ and $n$

$$
x^{n} \otimes y^{m}=(x \otimes y)^{n m}(y \otimes[x, y])^{n\left(\frac{m}{2}\right)}(x \otimes[x, y])^{m\left(\frac{n}{2}\right)}
$$


3. Main results. We proceed now with the main result of this paper, giving an estimate for the number of generators of $G \otimes G$ in terms of the number of generators of $G$, provided $\operatorname{cl}(G)=2$.

THEOREM 3.1. Let $G$ be a group of nilpotency class two with $d(G)=n$, then

$$
d(G \otimes G) \leq \frac{n\left(n^{2}+3 n-1\right)}{3}
$$

This theorem can be easily obtained using the lemmas of the preceding section. However, for use in our final theorem we need an explicit expression for each $g \otimes h$ in terms of the generators obtained in the above theorem. The proof of Theorem 3.1 will reflect this.

Proof of Theorem 3.1. Let $\left\{x_{1}, \ldots, x_{n}\right\}$ be a minimal generating set for the group $G$. We shall show that $G \otimes G$ is generated by all elements of the form $x_{i} \otimes x_{j}, 1 \leq i \leq n$, $1 \leq j \leq n, x_{i} \otimes\left[x_{j}, x_{k}\right], 1 \leq i \leq n, 1 \leq j<k \leq n$. Let $g, h \in G$. Then $g$ and $h$ can be represented as $g=U V$ and $h=U^{\prime} V^{\prime}$, where

$$
\begin{array}{rlrl}
U & =\prod_{i=1}^{n} x_{i}^{m_{i}}, & V & =\prod_{1 \leq j<k \leq n}\left[x_{j}, x_{k}\right]^{j_{j k},}, \\
U^{\prime} & =\prod_{i=1}^{n} x_{i}^{m_{i}^{\prime}}, \quad V^{\prime}=\prod_{1 \leq j<k \leq n}\left[x_{j}, x_{k}\right]^{l_{j k}},
\end{array}
$$

with integers $m_{i}, m_{i}^{\prime}, l_{j k}, l_{j k}^{\prime}$. Thus every generator $g \otimes h$ of $G \otimes G$ can be then written as $U V \otimes U^{\prime} V^{\prime}$. Since both $V$ and $V^{\prime}$ are in the center of the group, then

$$
U V \otimes U^{\prime} V^{\prime}=\left(U \otimes U^{\prime}\right)\left(U \otimes V^{\prime}\right)\left(V \otimes U^{\prime}\right)\left(V \otimes V^{\prime}\right) .
$$

Expanding $V \otimes V^{\prime}$ by Lemma 2.5 and observing Corollary 2.2, we obtain $V \otimes V^{\prime}=1_{\otimes}$. We expand the three remaining terms according to Lemmas 2.5 and 2.6. It follows easily that

$$
\left(U \otimes V^{\prime}\right)\left(V \otimes U^{\prime}\right)=\prod_{i=1}^{n} \prod_{1 \leq j<k \leq n}\left(x_{i} \otimes\left[x_{j}, x_{k}\right]\right)^{m_{i} i_{k}-m_{i} i_{k}}
$$

Expansion by Lemma 2.5 yields $U \otimes U^{\prime}=\prod_{i=1}^{n} \prod_{j=1}^{n}\left(x_{i}^{m_{i}} \otimes x_{j}^{m_{j}}\right) \cdot M$, where

$$
M=\prod_{i=1}^{n} \prod_{j=2}^{n} \prod_{k=1}^{j-1}\left(\left[x_{j}, x_{i}\right] \otimes x_{k}\right)^{m_{j} m_{k} m_{i}} \prod_{j=1}^{n} \prod_{i=2}^{n} \prod_{k=1}^{i-1}\left(x_{k} \otimes\left[x_{i}, x_{j}\right]\right)^{m_{k} m_{i} m_{j}^{\prime}} .
$$

By combining terms, applying Lemma 2.6 , and reindexing we can show that

$$
U \otimes U^{\prime}=\prod_{i=1}^{n} \prod_{j=1}^{n}\left(x_{i} \otimes x_{j}\right)^{m_{i} m_{j}^{\prime}} \prod_{i=1}^{n} \prod_{j=1}^{n}\left(x_{i} \otimes\left[x_{i}, x_{j}\right]\right)^{m_{j}^{\prime}\left(\frac{\left(i_{i}\right)-m_{j}\left(m_{2}^{\prime}\right)}{2}\right)}
$$

Thus the tensor square is generated by the desired elements.

If $n=2$, then $G \otimes G$ is generated by

$$
x_{1} \otimes x_{2}, x_{2} \otimes x_{1}, x_{1} \otimes x_{1}, x_{2} \otimes x_{2}, x_{1} \otimes\left[x_{1}, x_{2}\right], x_{2} \otimes\left[x_{1}, x_{2}\right] .
$$


Thus $d(G \otimes G) \leq 6=\frac{2}{3}(4+6-1)$, the desired result. Now let $n \geq 3$. There are $n^{2}$ generators of the form $x_{i} \otimes x_{j}$ and $n(n-1)$ generators of the form $x_{i} \otimes\left[x_{j}, x_{i}\right], i \neq j$. Initially there are $n(n-1)(n-2)$ generators of the form $x_{i} \otimes\left[x_{j}, x_{k}\right]$ for distinct $i, j, k$. Since $\left[x_{j}, x_{k}\right]=\left[x_{k}, x_{j}\right]^{-1}$ it follows from Proposition 3 in [3] that we can assume $j<k$. However by Corollary 2.4 we have $x_{i} \otimes\left[x_{j}, x_{k}\right]=\left(x_{j} \otimes\left[x_{i}, x_{k}\right]\right)\left(x_{k} \otimes\left[x_{j}, x_{i}\right]\right)$. Thus there are only $2\left(\begin{array}{c}n \\ 3\end{array}\right)=\frac{1}{3} n(n-1)(n-2)$ generators of the form $x_{i} \otimes\left[x_{j}, x_{k}\right]$ necessary, and therefore

$$
d(G \otimes G) \leq n^{2}+n(n-1)+\frac{1}{3} n(n-1)(n-2)=\frac{1}{3} n\left(n^{2}+3 n-1\right) .
$$

REMARK. It should be noted that the above can be extended to the case where $G$ is not finitely generated. In particular, $d(G \otimes G) \leq d(G)$ if $d(G)$ is an infinite cardinality.

Our next result shows that the estimate for $d(G \otimes G)$, as given in Theorem 3.1, is sharp. We will show that for $G=F_{n} / \gamma_{3}\left(F_{n}\right)$, the free nilpotent group of class 2 on $n$ generators, equality holds in Theorem 3.1. For $n=2$ this already has been shown in [1].

THEOREM 3.2. For any positive integer $n \geq 2$ the tensor square of a free $n$-generated nilpotent group of class 2 is free abelian of rank $\frac{1}{3} n\left(n^{2}+3 n-1\right)$.

Proof. By Theorem 3.1 we already have that the rank of this group is at most $\frac{1}{3} n\left(n^{2}+3 n-1\right)$. We now show that the rank is at least this number. For brevity set $\mathscr{H}_{n}=F_{n} / \gamma_{3}\left(F_{n}\right)$, and let $\mathscr{H}_{n}=\left\langle x_{1}, \ldots, x_{n}\right\rangle$. Every $g \in \mathscr{H}_{n}$ can be written as

$$
g=\prod_{1 \leq i \leq n} x_{i}^{m_{i}(g)} \cdot \prod_{1 \leq j<k \leq n}\left[x_{j}, x_{k}\right]^{l_{j k}(g)}
$$

with unique integers $m_{i}(g)$ and $l_{j k}(g)$. Thus there are well-defined functions $m_{i}$ for $1 \leq i \leq n$ and $l_{j k}$ for $1 \leq j<k \leq n$ from $\mathscr{H}_{n}$ to $\mathbb{Z}$.

We note that $m_{i}(g h)=m_{i}(g)+m_{i}(h), l_{j k}(g h)=l_{j k}(g)+l_{j k}(h)-m_{j}(h) m_{k}(g), m_{i}\left({ }^{g} h\right)=$ $m_{i}(h)$, and $l_{j k}\left({ }^{g} h\right)=l_{j k}(h)+m_{i}(g) m_{j}(h)-m_{i}(h) m_{j}(g)$. We define now functions $z_{i j}, z_{i i j}, z_{i j i}$, $z_{i j k}$, from $\mathscr{H}_{n} \times \mathscr{H}_{n}$ to $\mathbb{Z}$ by

$$
\begin{gathered}
z_{i j}(g, h)=m_{i}(g) m_{j}(h), \quad 1 \leq i \leq n, \quad 1 \leq j \leq n \\
z_{i j}(g, h)=m_{i}(g) l_{i j}(h)-m_{i}(h) l_{i j}(g)+m_{j}(h)\left(\begin{array}{c}
m_{i}(g) \\
2
\end{array}\right) \\
-m_{j}(g)\left(\begin{array}{c}
m_{i}(h) \\
2
\end{array}\right)+m_{i}(g) m_{i}(h)\left(m_{j}(h)-m_{j}(g)\right), \quad 1 \leq i<j \leq n, \\
z_{i j i}(g, h)=m_{i}(g) l_{j i}(h)-m_{i}(h) l_{j i}(g)-m_{j}(h)\left(\begin{array}{c}
m_{i}(g) \\
2
\end{array}\right) \\
+m_{j}(g)\left(\begin{array}{c}
m_{i}(h) \\
2
\end{array}\right), \quad 1 \leq j<i \leq n, \\
z_{i j k}(g, h)=m_{i}(g) l_{j k}(h)-m_{i}(h) l_{j k}(g)+m_{k}(h) l_{i j}(g)-m_{k}(g) l_{i j}(h) \\
+m_{i}(g) m_{j}(g) m_{k}(h)-m_{i}(h) m_{j}(h) m_{k}(g) \\
+m_{i}(h) m_{j}(g) m_{k}(h)-m_{i}(g) m_{j}(h) m_{k}(g), \quad 1 \leq i<j<k \leq n, \\
z_{i j k}(g, h)=m_{i}(g) l_{j k}(h)-m_{i}(h) l_{j k}(g)+m_{k}(g) l_{j i}(g) \\
-m_{k}(h) l_{j i}(h)+m_{j}(g) m_{i}(h) m_{k}(h)-m_{j}(h) m_{i}(g) m_{k}(g), \quad 1 \leq j<i<k \leq n .2
\end{gathered}
$$


To check that the $z_{*}$, where $z_{*}$ is one of the mappings defined in (3.2.1)-(3.2.5), is a crossed pairing, we have to verify by Definition 1.1 that

$$
z_{*}\left({ }^{g} g^{\prime}, g^{g} h\right)=z_{*}\left(g g^{\prime}, h\right)-z_{*}(g, h)=z_{*}\left(g^{\prime}, g h\right)-z_{*}\left(g^{\prime}, g\right) \text {. }
$$

It follows from Proposition 1.2 that each $z_{*}$ lifts to a homomorphism, and thus $\mathscr{H}_{n} \otimes \mathscr{H}_{n}$ has at least rank $\frac{1}{2} n\left(n^{2}+3 n-1\right)$, and the theorem is shown.

Acknowledgement. This paper is part of the author's Ph.D. dissertation at the State University of New York at Binghamton. The author would like to thank his advisor Professor Luise-Charlotte Kappe for her guidance and encouragement.

\section{REFERENCES} 95-106.

1. R. Aboughazi, Produit tensoriel du group d'Heisenberg, Bull. Soc. Math. France 115 (1987),

2. M. Bacon and L.-C. Kappe, The nonabelian tensor square of a 2-generator $p$-group of class 2, Arch. Math. (Basel) 61 (1993), 508-516.

3. R. Brown, D. L. Johnson, and E. F. Robertson, Some computations of nonabelian tensor products of groups, J. Algebra 111 (1987), 177-202.

4. R. Brown and J.-L. Loday, Excision homotopique en basse dimension, C.R. Acad. Sci. Paris Sér. I Math. 298 (1984), 353-356.

5. R. Brown and J.-L. Loday, Van Kampen theorems for diagrams of spaces, Topology 26 (1987), 311-335.

6. J. H. C. Whitehead, A certain exact sequence, Ann. of Math. 52 (1950), 51-110.

Department of Mathematics

BINGHAMTON UNIVERSITY

BinghamTON, NY 13902-6000, U.S.A.

Present address:

Department of Mathematics

UNIVERSITY OF WISCONSIN-PLATTEVILLE

Platteville, WI 53818, U.S.A. 